\title{
The gut and oral glucose tolerance
}

The oral glucose tolerance test has a long history, ${ }^{1}$ but has had from time to time to endure considerable criticism. One review of the test published 10 years ago reads almost like an obituary, pointing out that the considerable number of variables involved result in both poor reproducibility and in difficulties in interpretation. ${ }^{2}$ In spite of this the oral glucose tolerance test survives and for routine use in the diagnosis of diabetes mellitus it has not been replaced by the intravenous glucose tolerance test, which, although claimed to be more reproducible, is less convenient.

One advantage of using the intravenous glucose tolerance test is that it is unaffected by gastrointestinal factors such as gastric emptying rate and intestinal absorption. These variables are in themselves of considerable interest to gastroenterologists, and recent studies have made it possible to define in a little more detail their influence on glucose tolerance curves. The gastroenterologist's interest in oral glucose tolerance can also extend to the role of the liver, as all the glucose load has to pass through this organ before reaching the systemic circulation. The recent recognition that a gastrointestinal factor, released by the small intestine in response to oral glucose, plays an important role in the metabolism of absorbed glucose by stimulating insulin secretion ${ }^{3,4,5}$ has extended the known influence of the gut to include metabolism of glucose in muscle and adipose tissue, and has required a reappraisal of the differences between the results of oral and intravenous glucose tolerance tests. We can, therefore, legitimately include under the title of this survey more gastrointestinal 'variables' than were at one time thought necessary. Our list must include gastric emptying, intestinal absorption, the gastrointestinal hormonal stimulus to insulin release, the liver, and the pancreatic islets.

Variable gastric emptying has frequently been incriminated as a cause of the known poor reproducibility of oral glucose tolerance tests. ${ }^{2}$ There is no evidence to support these accusations against an organ whose emptying of a glucose solution has been shown by Hunt and his colleagues $^{6}$ to proceed in an orderly manner at a rate determined largely by its volume and osmolality. In the individual subject the rate is constant from day to day and is little affected by posture. ${ }^{7}$ Over a wide range of glucose concentrations the gastric emptying rate falls as the concentration of glucose rises. It has been suggested by Hunt that this is due to the stimulation of receptors in the duodenum sensitive to the osmotic pressure of the duodenal contents. If this was the case, then the glucose polymers of 'liquid glucose BP', advocated for use in glucose tolerance tests because they are less nauseating, might be expected to leave the stomach more rapidly than glucose itself, but blood glucose curves after 
'liquid glucose' or glucose itself are very $\operatorname{similar}^{8,9}$ in the few comparative studies that have been performed. This is probably because, except in patients with severe exocrine pancreatic insufficiency, ${ }^{10}$ hydrolysis of glucose polymers in the upper small intestine is so rapid that even starch solutions leave the stomach at the same rate as isocaloric glucose solutions. Pancreatic insufficiency is the only cause of defective glucose polymer digestion, as the final stage of starch digestion, which is the breakdown of maltose and iso-maltose by disaccharidases of the brush border, is not a rate-limiting step, ${ }^{11}$ and hereditary defects of these particular enzymes do not occur. 'Liquid glucose' is probably more palatable than an equivalent amount of glucose in a nonpolymerized form, and it has been used in large-scale population surveys of glucose tolerance. ${ }^{12}$ It may be useful if a 100 grams of glucose, widely used in the United States, is the preferred dose. The theoretical drawback that it may give misleading results in pancreatic insufficiency is perhaps insufficient to constitute a serious objection to its use.

In Great Britain, the usual dose of glucose employed for the oral test is 50 grams. Dissolved in $250 \mathrm{ml}$ of water this makes a very hypertonic solution, but recent studies of gastric emptying during oral glucose tolerance tests ${ }^{13}$ have shown that, as is found with a more dilute solution of the same volume, ${ }^{6}$ emptying proceeded in an exponential manner after a variable but usually short initial delay. Significant delay due to nausea was not encountered. The rate is conveniently expressed as the $T_{\frac{1}{2}}$, the time taken for half the initial meal to leave the stomach, and in normal individuals this lies between 40 and 80 minutes. This supports old observations that at the time the blood sugar returns to normal an appreciable amount of sugar may still be in the stomach.14

The simultaneous determination of gastric emptying and glucose tolerance enables the relationship between the two to be studied in some detail in individual patients. Flat glucose tolerance curves, venous blood sugar levels rising less than $5 \mathrm{mg}$ per $100 \mathrm{ml}$ are seen occasionally in subjects with a normal gastric emptying time ${ }^{15}$ and in subjects with a normal pattern of plasma insulin levels. This confirms that in healthy young adults, particularly if venous rather than capillary blood is sampled, a flat glucose tolerance curve can be a normal finding and is a result of rapid metabolism and not of either deficient absorption or slow gastric emptying. On the other hand, there is no doubt that in certain pathological conditions changes in the gastric emptying rate may significantly alter the shape of the glucose tolerance curves. This has been demonstrated directly only in hyper- and hypothyroidism, ${ }^{13}$ but it is possible that the more rapid emptying found in many patients with duodenal ulcer ${ }^{16}$ may contribute to the higher glucose levels reported in these patients 15 minutes after oral glucose. ${ }^{17}$ The high peak glucose often seen after a partial gastrectomy is also no doubt due to rapid emptying of the gastric remnant. Reactive hypoglycaemia after partial gastrectomy has been attributed to hyperinsulinaemia produced by this high peak glucose, ${ }^{18}$ but measurement of serum insulin levels does not 
reveal evidence of such a direct relationship..$^{19}$ Reactive hypoglycaemı of a comparable extent can be induced by glucose infused intravenously over a period of 30 to 45 minutes, ${ }^{19}$ although with this route of administration serum insulin levels are much lower. Some other explanation must, therefore, be found for postgastrectomy reactive hypoglycaemia. Hypoglycaemia in a fasting subject is normally prevented by hepatic glucose production. As the blood sugar rises after a glucose load hepatic glucose production stops. As the blood glucose subsequently falls, the liver again begins to produce glucose, and so a normal fasting blood sugar is maintained. Reactive hypoglycaemia in either normal subjects or in subjects with a partial gastrectomy might, therefore, be taken to be due to the failure of the liver to resume glucose production sufficiently rapidly. The normal exponential pattern of gastric emptying ${ }^{6}$ results in a very gradual decline of the rate at which glucose enters the intestine and this should provide ideal conditions for the liver gradually to resume glucose production. A partial gastrectomy disturbs this normal pattern and exponential emptying is no longer seen. In susceptible subjects this is presumably responsible for reactive hypoglycaemia, but prospective studies in which glucose tolerance and gastric emptying rates are measured before and after operation are needed to elucidate the relative roles of postoperative rapid gastric emptying, and of some metabolic predisposition to reactive hypoglycaemia.

The absorption of glucose by the small intestine is highly efficient. After ingestion of a concentrated solution, a combination of slow gastric emptying, ${ }^{6}$ dilution within the duodenum, ${ }^{20}$ and active peristalsis $^{21}$ ensure that within the jejunum the glucose solution no longer remains hypertonic. Intubation studies of the small intestine have shown that a $30 \mathrm{~cm}$ length of human jejunum is capable of absorbing $20 \mathrm{~g}$ of glucose in one hour. ${ }^{22}$ As $30 \mathrm{~cm}$ represents only one tenth of the small intestine, it is clear that absorption is rarely a rate-limiting factor affecting glucose tolerance, as the stomach will only release about this amount of a glucose load of $50 \mathrm{~g}$ into the duodenum during the first hour after ingestion. ${ }^{13}$ Small intestinal mucosal damage therefore has to be both severe and extensive if the glucose tolerance curve is to be significantly affected, and in practice these conditions are fulfilled only in idiopathic steatorrhoea. ${ }^{23}$ Of other conditions studied, diabetes mellitus can be singled out as the only condition in which jejunal glucose absorption is claimed to be more rapid than in normal subjects ${ }^{24}$ but it is doubtful whether the small change demonstrated would significantly affect the glucose tolerance test.

It has recently been clearly established that the insulin response to orally administered glucose is greater than that to glucose given intravenously. ${ }^{3}$, ${ }^{25}$ This additional stimulus to insulin release has a profound influence on the disposal of a glucose load and hence on glucose tolerance. A considerable body of evidence has now accumulated to support the original concept that a hormonal mechanism is involved. Of the hormones known to be present in the gastrointestinal tract, 
secretin, ${ }^{4,5}$ pancreozymin, ${ }^{5}$ gastrin, ${ }^{5}$ and glucagon ${ }^{26,27}$ have all been shown to be capable, under varying conditions, of stimulating insulin release. Which if any of these is most important under normal conditions is not yet clear, but the answer should be available with the perfection of techniques for immunoassay of these hormones in peripheral blood. Some of the difficulties involved are exemplified by those encountered in attempting to assess the role of glucagon. There is evidence that the glucagon secreted into portal blood after a large glucose load arises from the gut and not the pancreas. ${ }^{28}$ Furthermore, 'gut' glucagon may differ from 'pancreatic' glucagon in its chemical, biological, and immunological properties. ${ }^{28}$ The problem posed by the presence in plasma of two immunologically cross-reacting glucagon-like substances is therefore considerable. Claims that secretin ${ }^{29}$ is the agent involved are difficult to reconcile with the knowledge that in man, ${ }^{30}$ as in animals, intraduodenal glucose infusion has no effect on exocrine pancreatic secretion. Pancreozymin is an unlikely candidate for the same reason, although it may well be responsible for much of the insulin release which occurs in response to the oral administration of amino acids. ${ }^{5,28}$ In isolated preparations of pancreas, insulin secretion can be promoted by intestinal extracts which contain little if any of the currently known intestinal hormones. ${ }^{31}$ It may well be, therefore, that some as yet unidentified hormone is the responsible agent.

The liver, situated between the portal and systemic circulation, is in a position to influence oral glucose tolerance profoundly. Glucose administered orally is metabolized more rapidly than when given intravenously, ${ }^{32}$ and it has been assumed that this is due to the removal by the liver of most of the administered oral load. The demonstration of increased insulin secretion after oral glucose provides an alternative explanation for the superiority of oral over intravenous glucose tolerance. A variety of different experimental approaches have recently provided evidence which suggests that glucose removal by the liver has been overestimated. First, glucose infused into the portal vein of anaesthetized dogs results in arterial glucose levels similar to those after administration into a peripheral vein. ${ }^{33}$ Secondly, after an end-to-side portacaval anastomosis in human subjects peripheral blood glucose and insulin levels following oral glucose are no higher than before operation. ${ }^{\mathbf{1 5}}$ And thirdly, isotopic studies in unanaesthetized dogs indicate that about threequarters of a load of UC14 glucose given orally reaches the peripheral circulation. ${ }^{34}$ The cause of the impaired tolerance to both oral and intravenous glucose tolerance seen in the majority of patients with liver disease is therefore obscure, but it is certainly not simply a matter of a diminished mass of functioning tissue. Many possibilities have been considered and the subject has recently been fully reviewed. ${ }^{35}$ One factor is that cirrhotic patients are, rather like obese subjects, resistant to the action of both endogenous and exogenous insulin, but the cause of this insulin resistance is unknown. Fortunately, although of theoretical interest, the disturbed glucose metabolism is of little practical 
importance, as cirrhotic patients rarely develop symptoms of diabetes in spite of their abnormal glucose tolerance, and they do not become ketotic.

The pancreas is the last organ to concern us directly in this review. The basic biochemical abnormality in diabetes mellitus remains unknown, but most studies now agree that, except in obese subjects, defective insulin secretion is demonstrable in most diabetics of both 'juvenile' and 'maturity onset' varieties. In most diabetic subjects there is no exocrine pancreatic disease, and even the islets may be normal histologically. Chronic pancreatitis does, however, cause overt diabetes in some patients, and most patients with this condition have impaired insulin secretion ${ }^{36,37}$ even if this is not sufficiently severe to produce disturbances in carbohydrate tolerance. Ohlsén ${ }^{37}$ has shown some correlation between the degree of impairment of exocrine and endocrine pancreatic function in chronic pancreatitis, and has suggested that combined tests of endocrine and exocrine pancreatic function can contribute to the accurate diagnosis of these patients. The diagnostic value of serum insulin levels has also been suggested by Joffe et al..$^{36}$ They measured plasma insulin after intensive $\beta$-cell stimulation with oral glucose, tolbutamide, and glucagon, a procedure which can be compared with the maximal stimulus type of test used to study exocrine function of the stomach and pancreas. Under these conditions low insulin responses were found in all their patients with chronic pancreatitis. Insulin secretion has not been studied in patients with haemochromatosis, and the relative contribution made by pancreatic disease and cirrhosis to the impaired glucose tolerance seen in this condition is unknown. ${ }^{35}$ The diabetes is not caused simply by a diminished capacity for insulin secretion. Many cases require more insulin for their control than is the case in subjects with total pancreatectomy and occasionally severe insulin resistance is encountered, several hundred units daily being required for control. The diabetes of haemochromatosis is worthy of further study, not least because it is often considerably improved after treatment by repeated venesection. Whether this is due to improved liver function or to improvement in islet-cell function is not known.

The familiarity and ease of performance of the oral glucose tolerance test ensure that, carried out under careful standardized conditions, it will retain its important role in the diagnosis of diabetes mellitus. For the clinical investigator the test involves too many variables both in the gastrointestinal tract and elsewhere to be anything but the crudest of tools for the study of disturbed carbohydrate metabolism. To the gastroenterologist, its value will also remain strictly limited. A normal rise in blood glucose after administration of glucose mixed with the other monosaccharide component of a disaccharide has to be demonstrated before the finding of an absent or very small rise in blood sugar after administration of lactose or sucrose can be used as evidence for deficiency of the corresponding disaccharidase. Oral glucose tolerance 
curves can be of help in the diagnosis of pancreatic steatorrhoea and will continue to be useful in assessing symptoms which may be due to postgastrectomy hypoglycaemia.

C. D. HOLDSWORTH

\section{REFERENCES}

${ }^{1}$ McIntyre, N. (1967). Oral glucose tolerance: the role of the liver and small intestine. M.D. Thesis, University of London.

'Baird, J. D., and Duncan, L. J. P. (1959). The glucose tolerance test. Postgrad. med. J., 35, 308-314.

${ }^{3}$ McIntyre, N., Holdsworth, C. D., and Turner, D. S. (1964). New interpretation of oral glucose tolerance. Lancet, 2, 20-21.

'Dupré, J. (1964). An intestinal hormone affecting glucose disposal in man. Ibid, 2, 672-673.

'Unger, R. H., Ketterer, H., Dupré, J., and Eisentraut, A. M. (1967). The effects of secretin, pancreozymin, and gastrin on insulin and glucagon secretion in anesthetized dogs. J. clin. Invest., 46, 630-645.

'Hunt, J. N., and Knox, M. T. (1968). Regulation of gastric emptying. In Handbook of Physiology: Section 6, Alimentary Canal, edited by C. F. Code, Vol. 4, Chap. 94, p. 1917-1935. American Physiolog:cal Society, Washington, DC.

'Hunt, J. N., Knox, M. T., and Oginski, A. (1965). The effect of gravity on gastric emptying with various test meals. J. Physiol. (Lond.), 178, 92-97.

${ }^{8}$ Dodds, C., Fairweather, F. A., Miller, A. L., and Rose, C. F. M. (1959). Blood-sugar response of normal adults to dextrose, sucrose, and liquid glucose. Lancet, 1, 485-488.

'Butterfield, W. J. H., Sargeant, B. M., and Whichelow, M. J. (1964). The metabolism of human forearm tissues after ingestion of glucose, fructose, sucrose, or liquid glucose. Ibid, 1, 574-577.

${ }^{10}$ Mallinson, C. N. (1968). Effect of pancreatic insufficiency and intestinal lactase deficiency on the gastric emptying of starch and lactose (abstract). Gut, 9, 737.

"McMichael, H. B., Webb, J., and Dawson, A. M. (1967). The absorption of maltose and lactose in man. Clin. Sci., 33, 135-145.

${ }^{12}$ Butterfield, W. J. H. (1964). Summary of results of the Bedford diabetes survey. Proc. roy. Soc. Med., 57, 196-200.

${ }^{13}$ Holdsworth, C. D., and Besser, G. M. (1968). Influence of gastric emptying-rate and of insulin response on oral glucose tolerance in thyroid disease. Lancet, $2,700-702$.

${ }^{10 B e e l e r, ~ C ., ~ B r y a n, ~ A . ~ W ., ~ C a t h c a r t, ~ E . ~ P ., ~ a n d ~ F i t z, ~ R . ~(1922) . ~ A n ~ i m p r o v e d ~ a l i m e n t a r y ~ g l u c o s e ~ t o l e r a n c e ~ t e s t . ~}$ J. metab. Res., $1,549-560$.

${ }^{15}$ Holdsworth, C. D. (1968). Unpublished observations.

${ }^{10}$ Griffith, G. H., Owen, G. M., Campbell, H., and Shields, R. (1968). Gastric emptying in lealth and in gastroduodenal disease. Gastroenterology, 54, 1-7.

${ }^{17}$ Buchanan, K. D., McKiddie, M. T., Lindsay, A. C., and Manderson, W. G. (1967). Carbohydrate metabolism in duodenal ulcer patients. Gut, 8, 325-331.

${ }^{18}$ Gilbert. J. A. L., and Dunlop, D. M. (1947). Hypoglycaemia following partial gastrectomy. Brit. med. J., 2, 330-332.

${ }^{10}$ Holdsworth, C. D., McIntyre, N., and Turner, D. S. (1969). The pathophysiology of postgastrectomy reactive hypoglycaemia. Submitted for publication.

${ }^{20}$ Shay, H., Gershon-Cohen, J., Fels, S. S., and Munro, F. L. (1940). The fate of ingested glucose solutions of various concentrations at different levels of the small intestine. Amer. J. dig. Dis., 7, 456-462.

${ }^{21}$ Abbott, W. O., Karr, W. G., and Miller, T. G. (1938). Intubation studies of the human small intestine. VII. Factors concerned in absorption of glucose from the jejunum and ileum. Ibid, 4, 742-752.

${ }^{22}$ Holdsworth, C. D., and Dawson, A. M. (1964). The absorption of monosaccarides in man. Clin. Sci., 27, 371-379.

${ }^{25}$ Holdsworth, C. D., and Dawson, A. M. (1965). Glucose and fructose absorption in idiopathic steatorrhoea. Gut, 6, 387-391.

${ }^{24}$ Vinnik, I. E., Kern, F., Jr, and Sussman, K. E. (1965). The effect of diabetes mellitus and insulin on glucose absorption by the small intestine in man. J. Lab. clin. Med., 66, 131-136.

${ }^{25}$ McIntyre, N., Holdsworth, C. D., and Turner, D. S. (1965). Intestinal factors in the control of insulin secretion. J. clin. Endocr., 25, 1317-1324.

${ }^{20}$ Samols, E., Marri, G., and Marks, V. (1965). Promotion of insulin secretion by glucagon. Lancet, 2, 415-416.

${ }^{27}$ Turner, D. S., and McIntyre, N. (1966). Stimulation by glucagon of insulin release from rabbit pancreas in vitro. Ibid, 1, 351-352.

${ }^{20}$ Unger, R. H. (1968). New ideas concerning the physiologic roles of glucagon. Amer. J. med. Sci., 255, 273-276.

"Young, J. D., Lazarus, L., and Chisholm, D. J. (1968). Secretion and pancreozymin-cholecystokinin after glucose. Lancet, $2,914$.

soSum, P. T., and Preshaw, R. M. (1967). Intraduodenal glucose infusion and pancreatic secretion in man. Ibid, 2, 340-341.

${ }^{31}$ Turner, D. (1968). Incretin: an insulinotrophic hormone? (abstract), Diabetologia, 4, 177.

${ }^{32} \mathrm{Scow}, \mathrm{R}$. O., and Cornfield, J. (1954). Quantitative relations between the oral and intravenous glucose tolerance curves. Amer. J. Physiol., 179, 435-438.

${ }^{33}$ McIntyre, N., Turner, D. S., and Holdsworth, C. D. (1969). The role of the portal circulation in the hepatic uptake of glucose and fructose. Submitted for publication.

${ }^{34}$ Steele, R., Bjerknes, C., Rathgeb, I., and Altszuler, N. (1968). Glucose uptake and production during the oral glucose tolerance test. Diabetes, 17, 415-421.

s'Samols, E., and Holdsworth, C. D. (1968). Disturbances in carbohydrate metabolism: liver disease. In Carbohydrate Metabolism and its Disorders, p. 289-336. Edited by F. Dickens, P. J. Randle, and W. J. Whelan. Academic Press, London.

${ }^{36} \mathrm{~J}$ Jffe, B. I., Bank, S., Jackson, W. P. U., Keller, P., O'Reilly, I. G., and Vinik, A. I. (1968). Insulin reserve in patients with chronic pancreatitis. Lancet, $2,890-892$

${ }^{37}$ Ohlsén, P. (1968). Endocrine and exocrine pancreatic function in pancreatitis. Acta Med. Scand., Suppl. 484. 